\title{
Correction to: An Investigation on the Conjecture of Chen and Yi
}

\author{
Molla Basir Ahamed
}

\section{Correction to: Results Math (2019) 74:122 https://doi.org/10.1007/s00025-019-1045-4}

In the original publication, Examples 1.9 and 1.12 are exhibited inappropriately for infinite-order entire functions. Indeed, the author's main results have been proved for finite-order entire or meromorphic functions. The correct Examples 1.9 and 1.12 are provided in this correction.

Example 1. Let

$$
f(z)=\frac{a_{1} e^{2 i z}+a_{0} e^{-2 i z}}{b_{1} e^{2 i z}+b_{0}}, \quad \text { where } a_{1}, a_{0}, b_{1}, b_{0} \in \mathbb{C} \backslash\{0\} .
$$

Clearly $f$ is periodic, and also we see that $\mathcal{L}_{\pi}(f)=f$ when $c_{1}+c_{0}=1$.

Example 2. Let $f(z)=\frac{e^{z}+d^{2} e^{-z}}{2}, d$ is a nonzero complex constant, and for $c \neq k \pi i, k \in \mathbb{Z}$, let

$$
\mathcal{L}_{c}(f)=\frac{2 e^{c}}{e^{2 c}-1} f(z+c)-\frac{2}{e^{2 c}-1} f(z) .
$$

Clearly, $\mathcal{L}_{c}(f)=e^{z}$. We see that $f$ and $\mathcal{L}_{c}(f)$ share the values $a=d$ and $b=-d I M$ but $f$ neither has the specific form nor satisfies $\mathcal{L}_{c}(f) \equiv f$.

The next examples show that sharing two values $a, b C M$ cannot be replaced by one value $a C M$ and another value $b I M$ in our main result to get the conclusions.

The original article can be found online at https://doi.org/10.1007/s00025-019-1045-4. 
Example 3. Let $f(z)=1+\left(e^{z}-1\right)^{2}$ and $c \in \mathbb{C}$ be such that $e^{c}=-1$, let

$$
\mathcal{L}_{c}(f)=\frac{1}{4} f(z+c)-\frac{1}{4} f(z) .
$$

Clearly $\mathcal{L}_{c}(f)=e^{z}$. We see that $f$ and $\mathcal{L}_{c}(f)$ share the values $a=2 C M$ and $b=1 I M$, but note that $\mathrm{f}$ neither has the specific form nor satisfies $\mathcal{L}_{c}(f) \equiv f$.

Molla Basir Ahamed

Department of Mathematics

Kalipada Ghosh Tarai Mahavidyalaya

Bagdogra

West Bengal 734014

India

e-mail: basir_math_kgtm@yahoo.com ;

bsrhmd117@gmail.com

Publisher's Note Springer Nature remains neutral with regard to jurisdictional claims in published maps and institutional affiliations. 